\title{
Riscos ocupacionais e equipamentos de proteção individual em bombeiros da Brigada Militar
}

\author{
Occupational risks and personal protection equipments in firefighters
}

\author{
Bruna Zoehler Baumgarta, Andréia Barcellos Teixeira Macedob, Ana Paula Gossmann Bortolettia , \\ Sônia Beatriz Coccaro de Souzac
}

a Enfermeira. Escola de Enfermagem da Universidade Federal do Rio Grande do Sul (UFRGS)

${ }^{\mathrm{b}}$ Enfermeira. Mestre em Enfermagem pela UFRGS. Escola de Enfermagem da UFRGS.

c Enfermeira. Doutora em Psiquiatria pela UFRGS. Professora da Escola de Enfermagem da UFRGS.

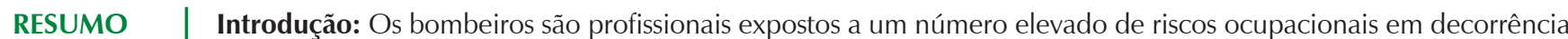
das múltiplas situações atendidas. Por este motivo, devem utilizar adequadamente os equipamentos de proteção individual (EPI), minimizando a exposição. Entende-se como risco ocupacional toda e qualquer possibilidade de que algum elemento ou circunstância existente, num dado processo e ambiente de trabalho, possa causar dano à saúde. Objetivo: Verificar a concordância entre a identificação correta dos riscos ocupacionais e a utilização do EPI.

Materiais e Métodos: Estudo transversal, com amostra de 83 bombeiros da Brigada Militar de Porto Alegre. O instrumento descreveu 13 ocorrências do cotidiano e os profissionais deveriam registrar riscos ocupacionais e EPI a ser utilizado. Para cada situação foi calculado o percentual de acertos para riscos e EPI e a concordância entre estes foi analisada através do método de Bland-Altman.

Resultados: A análise de concordância entre os percentuais das respostas corretas quanto ao uso do EPI e identificação dos riscos indicou que a utilização de EPI superestimou a identificação dos riscos, retratando que o número de acertos na identificação dos EPIs foi superior ao número de acertos na identificação dos riscos.

Conclusão: Observou-se que existe utilização do EPI na amostra, mas nem sempre é adequada ao risco ocupacional apresentado, trazendo vulnerabilidade para os profissionais durante o atendimento prestado.

Palavras-chave: saúde do trabalhador; riscos ocupacionais; condições de trabalho.

Introduction: Firefighters are professionals exposed to a high number of occupational risks, due to multiple situations. For this reason, they must properly use personal protective equipment (PPE), minimizing exposure. It is understood as occupational risk all and any possibility that some element or existing circumstance, in a given process or working environment, can cause damage to health.

Objective: To verify the correlation between the correct identification of occupational risks and the use of PPE.

Materials and Methods: Cross-sectional study with a sample of 83 firefighters of Brigada Militar in Porto Alegre. The instrument described 13 everyday occurrences and professionals should register occupational risks and PPE to their use. For each situation we calculated the percentage of correct answers to risks and PPE and the correlation between them was analyzed using the Bland-Altman method.

Results: The concordance between the percentage of correct answers on the use of PPE and risk identification indicated that the use of PPE overestimated the identification of risks, depicting the number of correct identification of PPE was higher than the number of hits in the identification of risks.

Conclusion: It was observed that there is use of PPE in the sample, but it is not always appropriate to the occupational risks, which brings vulnerability for professionals in the service provided.

Keywords: occupational health; occupational risks; working conditions. 


\section{INTRODUÇÃO}

A manutenção da saúde do trabalhador depende, entre outros fatores, da avaliação dos riscos ocupacionais a que estes profissionais estão expostos, e do investimento realizado pela empresa para minimizar ou extinguir os fatores de risco, tornando a atividade laboral mais segura. Entende-se como risco ocupacional toda e qualquer possibilidade de que algum elemento ou circunstância existente num dado processo e ambiente de trabalho possa causar dano à saúde"

Os riscos ocupacionais podem estar ocultos no ambiente de trabalho por falta de informação, situação em que o profissional trabalhador sequer suspeita da sua existência. Por outro lado, podem ser conhecidos, mas haver pouca valorização por parte dos profissionais por desconhecimento e falta de orientações adequadas. Podem se apresentar latentes, se manifestando e causando danos em situações de emergência ou condições de estresse; ou serem reais, conhecido de todos, porém, sem possibilidade de controle, principalmente em situações extremas, com exposições a eventos graves com risco de vida ${ }^{2}$.

Mapa de Risco é o instrumento capaz de reunir as informações necessárias para estabelecer o diagnóstico da situação de segurança e saúde no trabalho na empresa. De acordo com a Norma Regulamentadora (NR) de número 06, a empresa é obrigada a fornecer aos empregados, gratuitamente, Equipamentos de Proteção Individual (EPIs) adequados ao risco, em perfeito estado de conservação e funcionamento ${ }^{3,4}$.

Os bombeiros são profissionais expostos a um número elevado e variado de riscos ocupacionais em decorrência das múltiplas situações atendidas, sendo essas capazes de causar doenças laborais e acidentes de trabalho. Essas situações adversas são características do próprio trabalho, envolvendo atendimento de situações extremas, como longas horas no calor e fumaça, sem pausa para descanso. Dentre as atribuições destes profissionais, encontra-se a proteção contra incêndio, resgate e salvamento de pessoas e animais, remoção de fontes de perigo, atendimento a situações de incêndio e atuação em acidentes naturais, desastres ou envolvendo produtos classificados como perigosos à vida e saúde das pessoas 5 .

Os bombeiros devem se proteger dos riscos em suas atividades respeitando três condições básicas que constituem o triângulo da segurança: boa condição física e psíquica, equipamento de proteção individual adequado para cada situação e conhecimento e capacitação técnica. O EPI, para esta profissão, deve proteger principalmente, cabeça e pescoço, olhos, vias respiratórias, membros superiores e inferiores, cabendo ao empregador, nesta situação, adquirir o produto e orientar e supervisionar a utilização $0^{4,6}$. Entretanto, como membro da equipe de bombeiros estudada, uma das autoras evidenciou que a orientação aos novatos é passada pelos colegas mais antigos, não havendo um momento de orientação formal.

Empiricamente, acompanhando a atuação dos bombeiros da Brigada Militar de Porto Alegre, observa-se que os riscos ocupacionais mais frequentes são os biológicos, com a exposição a fluidos orgânicos e sangue; os físicos, produzido pelo ruído intenso, temperaturas extremas e mudanças climáticas; os ergonômicos, oriundos de posturas corporais inadequadas durante os atendimentos, manipulação de vítimas e equipamentos muito pesados; os químicos, pela manipulação de substâncias perigosas; os psicossociais, gerados por jornadas de trabalhos excessivas, fadiga e atendimento de situações traumáticas, extremas e estressantes.

Na última década, observa-se o crescente o interesse em pesquisas que avaliam as condições de trabalho, riscos ocupacionais e as patologias que afetam estes profissionais, no Brasil e no exterior ${ }^{7-10}$. Entretanto, ainda são pontuais os estudos que avaliam uso de EPIs como forma de proteção a determinados agravos de saúde, ponderando mais especificadamente os riscos biológicos ${ }^{11,12}$. E são escassos os estudos que avaliam a relação entre o reconhecimento do risco e a escolha do equipamento de proteção adequado, fato que motivou esta investigação.

O objetivo deste estudo foi verificar se os bombeiros identificam os riscos ocupacionais e se escolhem adequadamente o EPI em uma determinada situação. A hipótese foi de que os bombeiros utilizam os EPIs de acordo com os riscos ocupacionais por eles identificados no cotidiano laboral. A relevância do estudo reside no fato de descrever se os profissionais conseguem identificar os riscos aos quais estão expostos no seu cotidiano e de que forma estão se protegendo, visando oferecer informações para subsidiar projetos na área da saúde ocupacional para os trabalhadores.

\section{MATERIAIS E MÉTODOS}

Estudo de delineamento transversal, realizado nas sete Seções de Combate a Incêndio do Corpo de Bombeiros da Brigada Militar em Porto Alegre, no Rio Grande do Sul. A população foi constituída pelos 119 bombeiros que trabalhavam como combatentes nas sete estações, sendo que a seleção dos participantes foi intencional. O regime de revezamento de trabalho destes profissionais é de 24 horas de plantão para 72 horas de descanso.

Foram incluídos no estudo todos os profissionais que estavam trabalhando como combatentes no período da coleta dos dados e que tinham no mínimo dois anos de serviço no combate. Foram excluídos 36 profissionais que 
estavam em férias ou afastados, que não se encontravam de serviço nos dias das coletas, os soldados temporários e os que não aceitaram participar do estudo, totalizando amostra de 83 sujeitos.

A coleta dos dados ocorreu por meio de questionário autoaplicável elaborado pela autora. O instrumento apresentou 13 situações sobre ocorrências atendidas pelos bombeiros no cotidiano: 1) acidente veicular envolvendo um carro, no qual há uma vítima presa nas ferragens, com fratura exposta em membro inferior; 2) retirada de uma pessoa de dentro de um poço de água seco que possuí 10 metros de profundidade e 1,5 metro de diâmetro, com vítima consciente e apresentando fraturas em membros inferiores e escoriações; 3) gato em cima de uma árvore de médio porte; 4) salvamento de um cachorro que caiu dentro de um bueiro de 2 metros de profundidade; 5) corte de árvore que caiu devido a um temporal e encontra-se escorada no muro de uma residência; 6) vazamento de GLP (gás de cozinha) dentro de um apartamento; 7) extermínio de um enxame de abelhas localizado no forro de uma casa antiga, sendo que o único acesso é pelo telhado; 8) incêndio sem vítimas em uma casa de madeira que possui dois andares; 9) incêndio de vegetação em uma área íngreme e acidentada; 10) retirada de um corpo de uma pessoa que está dentro de um rio; 11) alagamento devido a chuvas, deixando famílias ilhadas, sem conseguirem sair de suas residências; 12) desabamento ocorrido em uma escavação subterrânea para esgoto, deixando três operários soterrados; 13) deslizamento de terra em terreno íngreme, com soterramento de algumas residências.

Em cada situação foi apresentada uma lista com os riscos ocupacionais e EPIs, conforme descritos na NR06, NR09 e também com os EPIs disponibilizados pela Corporação ${ }^{4,13}$. Diante de cada situação, os sujeitos foram convidados a assinalar quais riscos ocupacionais identificavam na situação proposta, bem como qual EPI utilizariam na respectiva situação.

Os dados descritivos foram sumarizados por meio da média, desvio padrão e frequências relativa e absoluta. A concordância entre as porcentagens na identificação correta dos riscos ocupacionais nas 13 situações e o adequado equipamento para proteção individual foi avaliada por meio da análise de concordância entre métodos de Bland-Altman. Este gráfico permite conhecer a relação das discordâncias com as medidas avaliadas ${ }^{15}$. Nesta análise, foram calculadas as médias das diferenças entre as porcentagens da identificação correta dos riscos ocupacionais e adequação do EPI. O teste t pareado foi utilizado para verificar se a diferença encontrada entre as médias era estatisticamente significativa, ou seja, diferente de zero, sendo também calculados os limites de concordância superiores e inferiores.
Os dados foram coletados após autorização do Comandante do Corpo de Bombeiros da Brigada Militar e aprovação do Comitê de Ética em Pesquisa da Universidade Federal do Rio Grande do Sul (UFRGS), com registro número 22150. Os bombeiros que aceitaram participar assinaram o Termo de Consentimento Livre e Esclarecido e receberam uma cópia deste documento. Foram preservados os princípios éticos de acordo com a Resolução 466/12, que trata das Diretrizes e Normas Regulamentadoras da Pesquisa Envolvendo Seres Humanos ${ }^{14}$.

\section{RESULTADOS}

Participaram do estudo 83 bombeiros, sendo 80 (96,4\%) do sexo masculino, $48(57,8 \%)$ com ensino médio completo, enquanto que 17 (20,5\%) estavam cursando ensino superior e $10(12 \%)$ já o haviam concluído. Quanto ao posto na Corporação, 22 (26,5\%) eram sargentos e 61 (73,5\%) soldados, com média de idade de $36,17 \pm 8,37$ anos.

A Tabela 1 demonstra o percentual de acertos em relação à identificação dos riscos ocupacionais nas situações pontuadas no instrumento, com média de $71,45 \pm 14,85 \%$. Observou-se que os profissionais apresentaram maiores percentuais de respostas corretas nos riscos relacionados à monotonia $(97,31 \%)$, trabalhos em turnos $(91,01 \%)$ e incêndio e explosão (91,01\%). Os riscos ocupacionais com maior percentual de erros foram os biológicos $(56,45 \%)$, posturas inadequadas $(54,95 \%)$ e poeiras $(46,43 \%)$.

Tabela 1. Percentual de acertos na identificação dos riscos ocupacionais nas situações do estudo.

\begin{tabular}{|c|c|c|c|}
\hline Grupo & Riscos & & $\%$ Acerto \\
\hline 1 & Físicos & $\begin{array}{l}\text { Ruído } \\
\text { Calor } \\
\text { Frio } \\
\text { Umidade }\end{array}$ & $\begin{array}{l}74,70 \\
87,30 \\
77,48 \\
67,47\end{array}$ \\
\hline 2 & Químicos & $\begin{array}{l}\text { Poeiras } \\
\text { Gases } \\
\text { Vapores }\end{array}$ & $\begin{array}{l}53,57 \\
57,28 \\
82,76\end{array}$ \\
\hline 3 & Biológicos & $\begin{array}{l}\text { Fungos, vírus, parasitas, } \\
\text { bactérias e protozoários }\end{array}$ & 43,55 \\
\hline 4 & Ergonômicos & $\begin{array}{l}\text { Levantamento e transporte } \\
\text { manual de peso } \\
\text { Monotonia } \\
\text { Repetitividade } \\
\text { Responsabilidade } \\
\text { Ritmo excessivo } \\
\text { Posturas inadequadas } \\
\text { Trabalho em turnos }\end{array}$ & $\begin{array}{l}68,86 \\
97,31 \\
74,79 \\
55,79 \\
72,29 \\
45,05 \\
91,01\end{array}$ \\
\hline 5 & Acidentes & $\begin{array}{l}\text { Arranjo físico inadequado } \\
\text { Iluminação inadequada } \\
\text { Incêndio e explosão } \\
\text { Eletricidade } \\
\text { Máquinas e equipamentos } \\
\text { sem proteção } \\
\text { Quedas } \\
\text { Animais peçonhentos }\end{array}$ & $\begin{array}{l}57,37 \\
74,24 \\
91,66 \\
77,11 \\
85,45 \\
71,92 \\
65,71\end{array}$ \\
\hline
\end{tabular}


O percentual de acertos em relação à identificação dos EPIs nas situações citadas no instrumento teve média de $79,55 \pm 7,47 \%$. Na Tabela 2 observa-se que nenhum EPI atingiu $100 \%$ de acerto e os menores percentuais ficaram em 66,08\%, em relação à escolha dos óculos de proteção, e $69,69 \%$ para o uso da bota. O maior percentual de acertos ficou na escolha da roupa de apicultor (99,54\%), seguido pela luva de raspa $(89,53 \%)$.

A análise de concordância entre o percentual das respostas corretas quanto ao uso adequado dos EPIs e identificação dos riscos ocupacionais nas 13 situações apresentadas, apontou um viés de $-13,75 \%$, com limites de concordância superior $(\mathrm{CSL})=-2,09$ e limites de concordância inferior $(\mathrm{CIL})=-25,41$

Tabela 2. Percentual de acertos de identificação dos EPIs nas situações do estudo.

\begin{tabular}{|c|c|}
\hline EPIs & $\%$ Acerto \\
\hline Capacete de combate & 74,98 \\
\hline Capa & 74,05 \\
\hline Calça & 72,75 \\
\hline Cadeirinha de salvamento & 79,15 \\
\hline Bota & 69,69 \\
\hline Luva de raspa & 89,53 \\
\hline Balaclava & 85,17 \\
\hline Capacete de salvamento & 73,59 \\
\hline Óculos de proteção & 66,08 \\
\hline Luva de procedimento & 84,34 \\
\hline Roupa de apicultor & 99,54 \\
\hline Aparelho de respiração autônoma & 85,54 \\
\hline
\end{tabular}

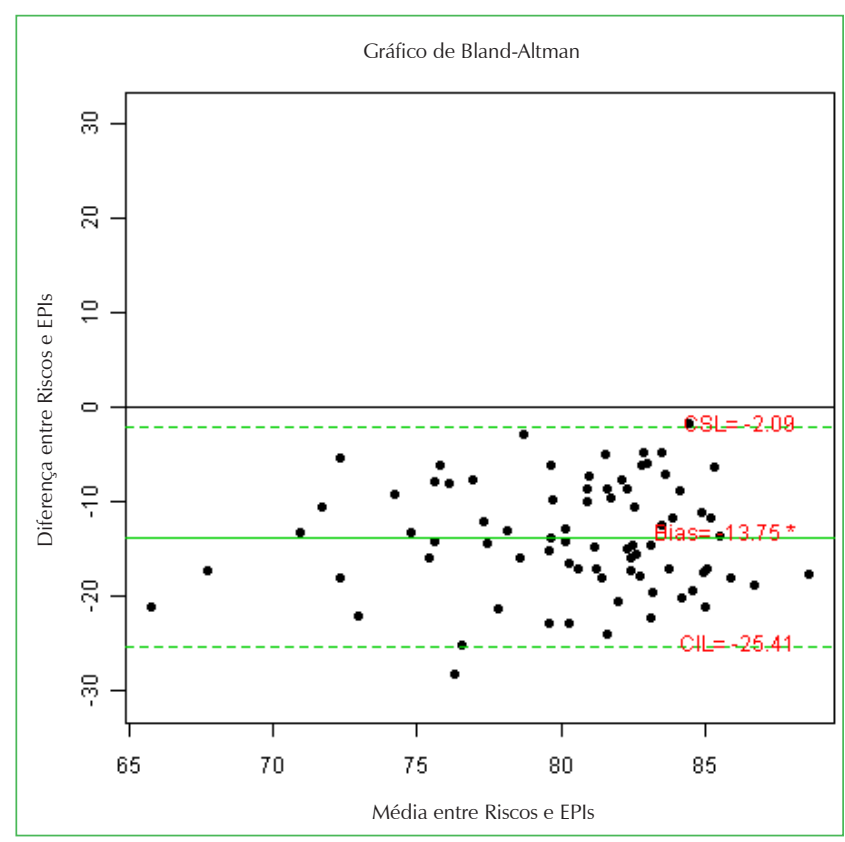

Figura 1. Resultado da análise da concordância entre percentual das respostas corretas quanto ao uso adequado dos EPIs. para $(\mathrm{t}=-21,49 ; \mathrm{gl}=82 ; p<0,001)$, indicando que a utilização de EPI superestimou a identificação dos riscos. Este achado indica que o número de acertos na identificação dos EPIs foi superior ao número de acertos na identificação dos riscos nas 13 situações. Este resultado encontra-se exposto na Figura 1.

\section{DISCUSSÃO}

A hipótese de que os bombeiros utilizam os EPIs de acordo com o reconhecimento dos riscos ocupacionais aos quais estão expostos no cotidiano laboral não foi comprovada. Identificou-se índice menor de reconhecimento dos riscos do que o esperado. Este dado sugere que a utilização dos EPIs não estaria ocorrendo com base num pensamento crítico sobre o tipo de proteção específica e sim devido a um comportamento reproduzido entre os pares.

Os bombeiros apresentaram média de acertos dos riscos ocupacionais de $71,45 \pm 14,85 \%$. A avaliação sobre a percepção do risco em diversas categorias profissionais demonstrou que a interpretação acaba sendo subjetiva e multifatorial, construída através da cultura organizacional, da experiência vivida no local de trabalho e de fatores pessoais. Por este motivo, alguns riscos são mais valorizados em detrimento de outros. A percepção do risco ainda é influenciada pelo setor em que o profissional atua, seu tempo de atuação profissional e seu nível de instrução ${ }^{16}$.

O achado de 56,45\% de erros no reconhecimento do risco biológico justifica a preocupação de alguns pesquisadores com o tema. Estudo realizado em Minas Gerais com militares do corpo de bombeiros demonstrou que 31,4\% dos profissionais não sabiam sobre o risco de aquisição de doenças pela exposição da mucosa ocular à matéria orgânica. Ainda, 32\% dos bombeiros não tinham conhecimento de como dar encaminhamento após acidente com resíduos biológicos. A exposição a material biológico pode ocasionar a contaminação por micro-organismos potencialmente causadores de infecções, o que, consequentemente, pode acarretar tanto ausências ao trabalho, temporárias ou permanentes, como licenças médicas e até mesmo casos de invalidez ${ }^{17}$. Por este motivo, o percentual de $31,4 \%$ é alto, tratando-se de risco iminente de transmissão e adoecimento.

Somando-se aos dados citados acima, encontramos $33,92 \%$ dos bombeiros que não associaram o risco biológico ao uso dos óculos de proteção. A literatura demonstra resultados de baixa adesão a este EPI em diferentes profissões. Estudo realizado com profissionais da enfermagem durante aspiração de vias aéreas, procedimento com alto risco de acidente com material biológico, identificou que nenhum dos 34 profissionais observados usou este EPI ${ }^{18}$. Em investigação realizada com a equipe multidisciplinar de atendimento pré- 
hospitalar constatou-se que apenas 58\% dos profissionais usavam rotineiramente os óculos de proteção ${ }^{19}$.

Observou-se também neste estudo certa desvalorização da poeira enquanto risco ocupacional, uma vez que não foi identificada em $46,43 \%$ das situações. Investigação realizada em bombeiros do Mato Grosso do Sul demonstrou que $83,4 \%$ dos profissionais não conseguem identificar os riscos químicos com poeira, gases e vapores ${ }^{20}$ na sua atividade profissional.

A média de acerto em relação ao uso do EPI foi de $79,55 \pm 7,47 \%$. Encontram-se valores diversos na literatura sobre o EPI, como uso incompleto em $46.7 \%$ entre bombeiros de Minas Gerais e $84 \%$ em Santa Catarina ${ }^{11,22}$. A NR06 dispõe que é de responsabilidade da empresa orientar e treinar o trabalhador sobre o uso adequado do $\mathrm{EPI}^{4}$. A média encontrada nos bombeiros de Porto Alegre pode ser considerada boa, visto que existem valores inferiores em outros estudos. Mas a utilização pela simples repetição de comportamento também não pode ser considerada ideal.

Além da falta de adesão ao EPI, outros fatores preocupantes têm sido apontados ultimamente. A mídia tem mostrado com frequência os problemas ocorridos pela quantidade e adequação dos equipamentos fornecidos aos profissionais, demonstrando que o risco maior de doença do trabalho pode não estar ligado somente à utilização ou não deste material. Estudo realizado com bombeiros do Mato Grosso do Sul demonstrou que 69\% dos acidentes de trabalho ocorreram por inadequação do material ${ }^{20}$. Já em Minas Gerais, os bombeiros verbalizaram dificuldade na utilização dos EPIs pela indisponibilidade do material na viatura que está atendendo a ocorrência, falta de tamanho adequado do material, material de qualidade ruim, equipamento de uso individual que acaba sendo de uso coletivo, entre outros problemas $^{17}$. Na percepção dos profissionais desta área, a insuficiência de recursos materiais e humanos, somada à falta de treinamentos técnicos de reciclagem são fatores estressantes que contribuem para diminuição da qualidade de vida no trabalho ${ }^{21}$.

A deficiência na percepção e/ou desconhecimento sobre os riscos aos quais estão suscetíveis sugere necessidade de reavaliar as atividades de educação continuada e permanente em biossegurança para o profissional bombeiro. Programas de educação, discussão de situações cotidianas e participação dos profissionais na resolução dos problemas podem contribuir para qualificação do trabalho, viabilizando não apenas a atualização do profissional, mas também a tomada de decisões e atitudes conscientes quanto à utilização do EPI. Cabe aqui sugerir uma reflexão de como estão sendo realizadas as capacitações para os profissionais do Corpo de Bombeiros da Brigada Militar de Porto Alegre. Outro fator importante é o acompanhamento contínuo e a supervisão dos profissionais no local de trabalho.

Entende-se que a falta de equipamentos para a utilização nas ocorrências cotidianas não pode ser a justificativa para a ausência de conhecimento sobre os riscos impostos pela profissão, fato que se comprovou neste estudo, com utilização dos EPIs sem o devido conhecimento do risco. Conhecer o risco pode minimizar a exposição através de atitudes mais seguras no trabalho. Destaca-se aqui o papel das lideranças na realização de momentos de troca de conhecimento, na conscientização dos trabalhadores, na orientação das situações diárias de trabalho, no acompanhamento das situações de não adesão ao uso do EPI e no estímulo na busca individual de conhecimento referente ao trabalho e às medidas de proteção.

Os dados revelaram que, apesar dos profissionais fazerem uso dos EPIs, a utilização não é feita de forma consciente, uma vez que o reconhecimento do risco não é realizado de forma direta com a identificação do EPIs específicos, ou seja, todos os EPIs são utilizados em todas as situações, inferindo desta forma que estes profissionais não reconhecem a severidade da exposição ocupacional a qual estão submetidos, tornando-se vulneráveis durante o atendimento prestado.

\section{REFERÊNCIAS}

1. Porto MFS. Análise de riscos nos locais de trabalho: conhecer para transformar. Cadernos de Saúde do Trabalhador. São Paulo: Kingraf; 2000.

2. Sêcco IAO, Robazzi MLCC, Gutierrez PR, Matsuo T. Acidentes de trabalho e riscos ocupacionais no dia-a-dia do trabalhador: desafio para a saúde do trabalhador. Espaç Saúde. 2002;4(1):68-81.

3. Portaria no 24 do Ministério do Trabalho e Emprego (BR), de 12 de julho de 2011. Comissão Interna de Prevenção de Acidentes. DOU, 13 jul. 2011.

4. Portaria no 292 do Ministério do Trabalho e Emprego (BR), de 08 de dezembro de 2011. Equipamento de proteção individual - EPI. DOU, 9 dez. 2011.

5. Forlin MAS. Riscos profissionais. Porto Alegre: Polost; 2005.

6. Francisco HS. Importância dos EPIs nas atividades de combate a incêndio. Revista Emergência RS [Internet]. abr. 2014 [acesso em: 08 jun. 2016]. Disponível em: http://www.revistaemergencia.com. br/noticias/leia_na_edicao_do_mes/artigo_trata_da_importancia_ dos_epis_nas_atividades_de_combate_a_incendio/ĀAjjA5y4/10313

7. Dias C, Slob, EMGB. Análise do processo saúde \& doença dos policiais militares do corpo de bombeiros de um município do Vale do Paraíba. Rev Eletrônica Enf Vale do Paraíba [Internet]. jun.jul. 2015 [acesso em: 01 jun. 2016];1(08): [14 telas]. Disponível em: http://www.publicacoes.fatea.br/index.php/reenvap/article/ viewArticle/1494

8. Melo LP. Síndrome de Burnout em bombeiros: prevalência, fatores de risco e proposta de intervenção [dissertação]. Porto Alegre: Pontifícia Universidade Católica do Rio Grande do Sul; 2015. 
9. Santos M, Imeida A. Principais riscos e fatores de risco ocupacionais associados aos bombeiros, eventuais doenças profissionais e medidas de proteção recomendadas. Rev Port Saúde Ocup [Internet]. 2016. [acesso em 01 jun. 2016]. Disponível em: http://www.rpso. pt/principais-riscos-e-fatores-de-risco-ocupacionais-associadosaos-bombeiros-eventuais-doencas-profissionais-e-medidas-deprotecao-recomendadas/

10. Spacassassi F. Avaliação hemodinâmica, estresse, perfil metabólico e balanço autonômico em profissionais bombeiros militares [dissertação]. São Paulo: Programa de mestrado em Medicina da Universidade Nove de Julho; 2015.

11. Oliveira AC, Machado BCA, Gama CS. Acidente ocupacional envolvendo material biológico entre bombeiros militares de Minas Gerais. Ciênc Cuid Saúde. 2014;13(2):343-349.

12. Torres MC, Gusmão CMP, Lúcio MG. Riscos Ocupacionais do atendimento pré-hospitalar: uma revisão bibliográfica. Interfaces Cient Saúde Ambiente. 2013;1(3):69-77. https://doi.org/10.17564/ 2316-3798.2013v1n3p69-77

13. Portaria MTE no 1.471, de 24 de setembro de 2014. NR 9 programa de prevenção de riscos ambientais. DOU, 25 set. 2014.

14. Resolução no 466 do Conselho Nacional de Saúde, de 12 de dezembro de 2012 (BR). Pesquisa envolvendo seres humanos. DOU, 13 jul. 2013.

15. Hirakata VN, Camey SA. Análise de concordância entre métodos de Bland-Altman. Rev HCPA [Internet]. 2009 [acesso em 26 maio 2015]; 29(3):[8 telas]. Disponível em: http://www.lume.ufrgs.br/ bitstream/handle/10183/28980/000738092.pdf?sequence $=1$
16. Areosa J. As perceções de riscos ocupacionais no setor ferroviário. Sociologia. 2014;75:83-107. https://doi.org/10.7458/ spp2014753577

17. Oliveira AC, Machado BCA, Gama CS. Conhecimento e adesão às recomendações de biossegurança no Corpo de Bombeiros Militar de Minas Gerais. Rev Esc Enferm USP. 2013;47(1):115-27. http:// dx.doi.org/10.1590/S0080-62342013000100015

18. Frota OP, Loureiro MDR, Ferreira. Aspiração endotraqueal por sistema aberto: práticas de profissionais de enfermagem em terapia intensiva. Esc Anna Nery. 2014;18(2):296-302.

19. Gomes BB, Santos LS. Acidentes laborais entre equipe de atendimento pré-hospitalar móvel (bombeiros/Samu) com destaque ao risco biológico. Revisa. 2012;1(1):40-9.

20. Contrera-Moreno L. Condições associadas à ocorrência de doenças infecciosas no trabalho operacional de bombeiros de Campo Grande, MS [dissertação]. Universidade Federal do Mato Grosso do Sul; 2012.

21. Monteiro JK, Maus D, Machado FR, Pesenti C, Bottega D, Carniel LB. Bombeiros: um olhar sobre a qualidade de vida no trabalho. Psicol Cienc Prof. 2007;27(3):554-65. https://doi.org/10.1590/ S1414-98932007000300014

22. Fermino IC. Biossegurança no atendimento pré- hospitalar nos quartéis de bombeiro militar das cidades de Armazém, Capivari de Baixo, Braço do Norte e Tubarão [monografia]. Florianópolis: UFSC; 2013. 